\title{
Spheres of Influence and Strategic Advocacy for Equity in Medicine
}

\author{
Kyle Karches, MD, MA ${ }^{7,2}$, Matthew DeCamp, $M D, P h D^{3,4}$, Maura George, $M D^{5}$, \\ Micah Prochaska, MD, MSC ${ }^{6,7}$, Milda Saunders, MD, MPH ${ }^{6,7}$, Bjorg Thorsteinsdottir, $\mathrm{MD}^{8,9}$, \\ and Elizabeth Dzeng, $M D, P h D, M P H^{10,11}$ (it)
}

\begin{abstract}
'Department of Internal Medicine, Saint Louis University, Saint Louis, MO, USA; ${ }^{2}$ Gnaegi Center for Health Care Ethics, Saint Louis University, Saint Louis, MO, USA; ${ }^{3}$ Division of General Internal Medicine, Department of Medicine, University of Colorado, Denver, CO, USA; ${ }^{4} \mathrm{Center}$ for Bioethics and Humanities, University of Colorado, Denver, CO, USA; ${ }^{5}$ Division of General Medicine, Emory University, Atlanta, GA, USA; ${ }^{6}$ Department of Medicine, Pritzker School of Medicine, The University of Chicago, Chicago, IL, USA; ${ }^{7}$ MacLean Center for Clinical Medical Ethics, The University of Chicago, Chicago, IL, USA; ${ }^{8}$ Division of Community Internal Medicine, Department of Medicine, Mayo Clinic, Rochester, MN, USA; ${ }^{9}$ Program in Bioethics, Mayo Clinic, Rochester, MN, USA; ${ }^{10}$ Division of Hospital Medicine, Department of Medicine, University of California, San Francisco, San Francisco, CA, USA; " Cicely Saunders Institute, King's College London, London, UK.
\end{abstract}

As the extent of health disparities in the USA has been revealed, particularly during the COVID-19 pandemic, physicians have increasingly attended to their roles as advocates for their patients and communities. This article presents "spheres of influence" as a concept that can help physicians think strategically about how to build upon their clinical work and expertise to promote equity in medicine. The physician's primary sphere of influence is in direct patient care. However, physicians today often have many other roles, especially within larger health care institutions in which physicians often occupy positions of authority. Physicians are therefore well-positioned to act within these spheres in ways that draw upon the ethical principles that guide patient care and contribute materially to the cause of equity for colleagues and patients alike. By making changes to the ways they already work within their clinical spaces, institutional leadership roles, and wider communities, physicians can counteract the structural problems that undermine the health of the patients they serve.

J Gen Intern Med 36(11):3537-40

DOI: $10.1007 / \mathrm{s} 11606-021-06893-4$

(c) Society of General Internal Medicine 2021

\section{INTRODUCTION}

Disparities laid bare by the COVID-19 pandemic and increasing recognition of systemic racism following the killing of George Floyd and others have galvanized physicians to advocate for their patients and community beyond the clinical setting. Many physicians participated in efforts such as "White Coats for Black Lives" in their communities and on social media to raise awareness of these issues. Although these symbolic gestures of solidarity are important, physicians as

Prior presentations: Not applicable.

Received February 10, 2021

Accepted May 3, 2021

Published online May 19, 2021 leaders within their institutions and communities also have a responsibility to promote and enact substantive changes that materially benefit the communities they serve.

The concept of "spheres of influence" is helpful for physicians to think strategically about how to use their influence and authority without encroaching on territories better addressed by other experts or medicalizing social goods that require a broader societal or political cooperation to achieve. ${ }^{1}$ In this paper, we apply this theory to describe practical ways that physicians can counteract health disparities. We explain how clinicians might draw upon their medical expertise and ethical principles to understand their sphere of influence and to advocate for structural changes that they are most empowered to enact. Clinicians can reduce health disparities by directly influencing policies within their own practices and institutions and by contributing in unique ways to local, regional, and national political processes.

\section{SPHERES OF INFLUENCE}

Spheres of influence refers to the concept that there are domains that individuals or institutions have relatively more influence over, and hence responsibility for, than others. Clinicians' quintessential core sphere of influence is in direct patient care, though, broadly speaking, generalist physicians occupy many diverse roles in society, including in research, education, and health policy. In this piece, we focus on the practicing clinician's sphere of influence. Patients seek the knowledge, advice, and expertise of clinicians, who in turn have a responsibility within this sphere to influence patients while adhering to the widely accepted ethical principles of beneficence, non-maleficence, patient autonomy, and justice. ${ }^{2}$

While direct patient care may be a clinician's core sphere, limiting physicians' influence to the bedside ignores their potential to influence care beyond the immediate clinical encounter. Clinicians also have spheres of influence within the care team, the health care institutions where they work, and 
as members of society. Physicians as leaders of care teams are influential in shaping the decisions and processes that affect their colleagues and co-workers. Within health care institutions, they are in positions to influence policies that affect not only patients but also the broader health care workforce. And as members of society, they along with public health experts are uniquely positioned to understand and explain how global social, political, and environmental forces together affect patients' health.

Clinicians may feel ethically obliged to act outside of their "core," but this extension carries ethical risks. Trust in the medical profession relies on patients' and the public's understanding of medicine's fundamental values. If members of the medical community go too far in attempting to exert influence beyond their expertise, they risk undermining that trust. For example, it is one thing to make known the health effects of climate change as a medical professional; it is quite another to appropriate trust in the medical profession to advocate for a carbon tax policy.

In the remainder of this paper, we provide practical examples of how physicians might consider exerting influence outside of patient care while remaining aligned with medicine's core values.

\section{SPHERES OF INFLUENCE AT THE HEALTH SYSTEMS LEVEL}

Clinicians have a unique opportunity to address health disparities by making important changes within their personal and professional practices. By making strategic efforts within these spheres of influence, the spaces where they work every day and often have significant authority, physicians can work to reduce inequity. Obviously, clinicians' most immediate sphere of influence is their daily interaction with patients. One way for clinicians to avoid perpetuating bias in these encounters is to learn about implicit bias and systemic racism. This work of education and reflection can help clinicians examine how their own beliefs and institutional structures might reflect and perpetuate the pervasive inequities in our society, leading them to make concrete changes to the way they practice medicine and advocate within their institutions. ${ }^{3}$

Physicians should also enact changes in the infrastructure of their practices to address disparities directly. ${ }^{4}$ While physicians have long recognized the importance of incorporating social determinants of health into their practices, implementing system-wide screenings in electronic medical records that help connect patients with available resources will improve clinicians' ability to care for a population with diverse needs. Standardizing questions such as "Have you been able to get everything you need to manage your health, including groceries, medications, and transportation services?" might lead to frank discussions about race and class in America, which clinicians today should be willing to engage. In order to respond appropriately, clinicians may need to advocate within their institutions for adequate financial support for staff such as social workers and case managers who are trained to help patients with these needs. ${ }^{5}$ They should also ensure that their practices utilize federally mandated interpreter services consistently and effectively to improve access for underserved communities. ${ }^{6}$ In addition, they should advocate to change or remove algorithms in health records that reinforce discrimination by erroneously incorporating race as a surrogate for other markers. $^{7}$

In their documentation of clinical encounters, clinicians should use neutral descriptive phrases instead of stigmatizing language, which biases clinicians to potentially unfair negative perceptions of the patient. The "drug seeking patient" may have a different evaluation and treatment plan from the "patient with uncontrolled pain." The "non-compliant patient" will be viewed more harshly than will "the patient who could not afford medications". 8

Clinicians' sphere of influence also includes supporting their colleagues, both personally and professionally. On a personal level, physicians should recognize the struggles facing colleagues from underrepresented groups who may have been asked by their institutions to speak about their personal experiences of racism and might feel pressured to provide solutions. They might come from communities suffering disproportionately from violence, discrimination, and disease, including COVID-19. Asking how they are doing, offering support, and shouldering what additional responsibilities can be offloaded further inclusivity and partnership.

In the professional realm, clinicians frequently work within institutions that have mission statements and advertisements that profess their commitments to diversity, equity, and inclusion. As revenue-generating and therefore empowered members of these institutions, clinicians can hold employers accountable for these promises in several ways. Physicians should serve as allies for colleagues by amplifying their voices, including the opinions of front-line staff such as front desk workers and medical assistants, around strategies to address health inequities. Everyone who contributes to these efforts, especially lower paid health care workers, should be compensated appropriately for their participation. Clinicians might also encourage their institutions to dedicate resources to promoting and hiring a diverse workforce and recommend colleagues from diverse and underrepresented backgrounds for leadership positions and invited presentations. ${ }^{9}$

Recognizing the impact of social determinants such as financial resources on health outcomes, physicians ought to advocate for a living wage for all employees within their institutions. Hospitals and health care systems are often anchor institutions within their communities, with many local employees from various racial and socioeconomic backgrounds, so promoting equity within these institutions is one important step toward addressing local health disparities. ${ }^{10}$

The Affordable Care Act requires non-profit hospitals to conduct regular Community Health Needs Assessments (CHNAs), giving clinicians who work at such facilities an 
additional opportunity to address local health disparities. ${ }^{11}$ Since the CHNA process requires hospitals to solicit input from local community representatives, clinician leaders could create permanent community advisory boards to work with local health departments and help direct institutional resources toward the community's actual needs. ${ }^{12}$ They might also consider more creative ways for their institutions to support and promote equity in their neighborhoods, ranging from mortgage loans that encourage staff to live nearby to bus lines that integrate the hospital with the local community. Although COVID-19 has placed financial strain on health care systems, the disproportionate toll of COVID-19 on people of color makes these efforts to counter structural racism more urgent now than ever.

Individual physicians within large bureaucratic institutions might have concerns that such advocacy would jeopardize their positions. Mechanisms for collective action can empower clinicians to stand in solidarity with their fellow institutional employees as they advocate for change. At some health centers, unions have been created to address this need. ${ }^{13}$ Professional societies such as the Society of General Internal Medicine (SGIM) also provide the necessary foundation for these efforts. Such groups can provide the support necessary for physician leaders in health care institutions to implement changes that address health disparities.

\section{SPHERES OF INFLUENCE AT THE LOCAL COMMUNITY AND NATIONAL LEVEL}

Clinicians witness the ways that local and national policies and patterns of behavior affect health outcomes, giving them a sphere of influence and an opportunity to advocate at this level. Many physicians have been powerful advocates for their patients in this way, at times taking personal risks to point out harmful practices or resist powerful lobbies. Recent examples include Dr. Mona Hanna-Attisha's crucial role in uncovering the Flint water crisis and physicians' outcry about the health effects of the policy of family separation at the US border. ${ }^{14,15}$ Even in the absence of such crises, social problems contribute to ill health and premature death, and clinicians are wellpositioned to advocate for change. ${ }^{16}$

Physicians commonly have access to leaders and opportunities to be involved in local policymaking. In these roles, they can also work to mitigate health disparities and structural discrimination. For instance, during the COVID-19 pandemic, local and regional leaders formed task forces to monitor outcomes and create protocols for allocation of scarce resources such as ventilators and vaccines. Clinician members of such task forces should work to ensure that these protocols do not further widen disparities. ${ }^{17}$ Physicians can also contribute uniquely to state and local political efforts to expand services such as Medicaid by explaining the ways these policy decisions affect their daily work with patients. In so doing, they should invite the participation of those directly affected by these policies, perhaps by using community engaged participatory research methods to ensure their work addresses the most pressing needs of their local communities. ${ }^{18}$ Lessons learned can be applied to both practice innovation and academic problem solving for patient and community benefit.

Clinicians should consider joining existing organizations and lobbies who are already working in their areas of interest, advocating alongside or led by the people whose interest they are fighting for. By joining political organizations and initiatives championing diversity and social justice, they can lend expertise to research and present compelling data and stories to support the cause. Physicians are also able to exert influence within their own organizations to promote policies that benefit the disadvantaged. Societies such as SGIM and the American College of Physicians (ACP) have created entities to address equity and inclusion and releasing public announcements and position papers calling for more just and equitable policies. When appropriate, professional organizations should also acknowledge and respond to past injustices in which they have been complicit. A recent example is the American Medical Association's (AMA) acknowledgment of its past discriminatory practices. ${ }^{19}$

\section{CONCLUSION}

In this paper, we outline practical ways physicians can work within their spheres of influence from their own practices to their wider communities to counteract disparities. We contend that physicians should draw upon their own expertise and ethical commitments to maximize the benefits of our interventions and avoid encroaching on territory better addressed by others, thereby potentially squandering our moral authority. Given the many ways systemic problems such as race and class-based discrimination confound our efforts to care for our patients, physicians should consider such advocacy an essential part of their professional duty. In solidarity with one another and with their patients, physicians can promote reforms in their institutions and communities that will enable them to improve the health of all patients.

\section{Acknowledgements:}

Contributors: The development of this manuscript was supported by the Society of General Internal Medicine (SGIM) Ethics Committee. We wish to acknowledge the members and staff of the SGIM Ethics Committee.

Corresponding Author: Elizabeth Dzeng, MD, PhD, MPH; Division of Hospital Medicine, Department of Medicine, University of California, San Francisco, San Francisco, CA, USA (e-mail: liz.dzeng@ucsf.edu).

\section{Declarations:}

Conflict of Interest: The authors declare that they do not have a conflict of interest. 
Disclaimer: The views expressed in this manuscript are the authors' own and do not represent the official policies of SGIM.

\section{REFERENCES}

1. DeCamp M, DeSalvo K, and Dzeng E. Ethics and spheres of influence in addressing social determinants of health. J Gen Intern Med 2020;35:2743-2745.

2. Beauchamp TL and Childress JF. Principles of Biomedical Ethics, 8 th ed. Oxford: Oxford University Press, 2019.

3. Maina IW, Belton TD, Ginzberg S, et al. A decade of studying implicit racial/ethnic bias in healthcare providers using the implicit association test. Soc Sci Med 2018; 199:219-229.

4. Griffith DM, Mason M, Yonas $\mathbf{M}$, et al. Dismantling institutional racism: theory and action. Am J Community Psychol 2007;39:381-392.

5. Buitron de la Vega P, Losi S, Martinez LS, et al. Implementing an EHRbased screening and referral system to address social determinants of health in primary care. Med Care 2019;57:S133-S139.

6. Karliner LS, Jacobs EA, Chen AH, and Mutha $\mathbf{S}$. Do professional interpreters improve clinical care for patients with limited English proficiency? A systematic review of the literature. Health Serv Res 2007;42(2):727-754.

7. Vyas DA, Eisenstein LG, and Jones DS. Hidden in plain sight reconsidering the use of race correction in clinical algorithms. NEJM 2020;383:874-882.

8. Goddu AP, O'Conor KJ, Lanzkron S, et al. Do words matter? Stigmatizing language and the transmission of bias in the medical record. J Gen Intern Med 2018;33(5):685-691.

9. Jackson CS, Gracia JN. Addressing health and health-care disparities: the role of a diverse workforce and the social determinants of health. Public Health Rep 2014;129:59-61.
10. Bindman A. A healthy living wage. JAMA 2015;314(21):2224-2225

11. Community health needs assessment for charitable hospital organizations - section 501(r)(3). IRS.gov. Updated 21 Aug 2020. Available from < https://www.irs.gov/charities-non-profits/community-health-needs-assessment-for-charitable-hospital-organizations-section-501r3>.

12. Ahmed SM, Young SN, DeFino MC, et al. Towards a practical model for community engagement: advancing the art and science in community health centers. J Clin Transl Sci 2017;1(5):310-315.

13. Thompson SL and Salmon JW. Physician strikes. Chest 2014;146(5):1369-1374.

14. Hanna-Attisha $\mathbf{M}$. What the eyes don't see: a story of crisis, resistance, and hope in an American city. New York: One World, 2019.

15. Miller D. AAP a leading voice against separating children, parents at border. AAP News. 14 Jun 2018.

16. Fried JE, Shipman SA, Sessums LL. Advocacy: achieving physician competency. J Gen Intern Med 2019;34(11):2297-2298.

17. Essien UR, Eneanya MD, Crews DC. Prioritizing equity in a time of scarcity: the COVID-19 pandemic. J Gen Intern Med 2020;35(9):27602762.

18. Israel BA, Coombe $\mathbf{C M}$, Cheezum RR, et al. Community-based participatory research: A capacity-building approach for policy advocacy aimed at eliminating health disparities. Am J Public Health. 2010;100(11):2094-2102.https://doi.org/10.2105/AJPH.2009.170506.

19. Madara JL. Reckoning with medicine's history of racism. 17 Feb 2021. Available from <https://www.ama-assn.org/about/leadership/reckoning-medicine-s-history-racism>.

Publisher's Note: Springer Nature remains neutral with regard to jurisdictional claims in published maps and institutional affiliations. 